\title{
Implementing Ad Hoc to Terrestrial Network Gateways
}

\author{
Jonathan McGee, Manish Karir, and John S. Baras \\ Center for Satellite and Hybrid Communication Networks \\ Department of Electrical and Computer Engineering \\ University of Maryland, College Park, MD 20742, USA \\ \{mcgee, karir, baras\}@isr.umd.edu
}

\begin{abstract}
In this paper we describe our experience of implementing a gateway between ad hoc and terrestrial routing protocols. Our implementation of the gateway includes support for both a unicast routing protocol as well as a multicast routing protocol. Though we limit our implementation to a particular set of protocols, we believe that the principles involved can easily be applied to other routing protocols. In particular, in this paper we detail our work on implementing a gateway between a network running MOSPF on a wired terrestrial network interface and MAODV on a wireless ad hoc network interface. Although we focus primarily on the single gateway scenario, we also discuss complications that arise from the use of multiple gateways and illustrate the potential failures that can arise in those scenarios.
\end{abstract}

\section{Introduction}

While there has been a lot of work on ad hoc routing protocols, the operation of a gateway between ad hoc and terrestrial domains has received relatively little attention. There is an increasing interest in this problem as people are gradually realizing that ad hoc networks probably will not operate as stand alone networks. There will be the need at some point to connect back to a terrestrial network.

There are various problems associated with building efficient routing protocol gateways between ad hoc and terrestrial networks. The core of the problem arises from the very different characteristics of these networks. Wired terrestrial networks are considered relatively stable in topology, therefore, routing protocols that have evolved to run in those environments are largely based on a proactive approach of maintaining routing information. Ad hoc networks, on the other hand, are more suited to use reactive approaches although proactive approaches to routing in ad hoc networks also exist. Reactive approaches are best suited for ad hoc networks because of the expected mobility of the nodes within these networks. A proactive approach would simply generate too much control overhead with even moderate mobility and network size. Hybrid approaches that attempt to optimize these two contrasting approaches have also been developed for ad hoc networks.

The problem of developing a gateway between a proactive terrestrial routing protocol and a proactive ad hoc network routing protocol is relatively simple as the routing protocols are similar in nature. Each protocol aims at constructing a detailed routing table that reflects the connectivity of the entire network and the gateway is then simply 
responsible for exporting and importing routing information from each protocol. This problem is similar in nature to the exchange of routing information between OSPF and BGP in terrestrial networks, for example.

The exchange of information between a proactive terrestrial routing protocol and a reactive ad hoc network routing protocol is a bit more challenging as the type of information that a proactive routing protocol requires is not the same as the information that a reactive ad hoc routing protocol makes available. Therefore, to achieve interoperation between the two requires implementing either complex registration/deregistration for ad hoc network nodes or developing a proxy routing daemon that can provide abstracted information on behalf of the ad hoc network to the terrestrial routing protocol.

In this paper we take the second approach where the gateway provides abstracted ad hoc network information to the terrestrial routing protocol. We address both the cases of unicast as well as multicast routing protocols. To demonstrate our approach we implemented our prototype solution using MOSPF as the terrestrial routing protocol and MAODV as the ad hoc network routing protocol. Our approach does have the drawback that details of node location and state within the ad hoc network are lost. This can create problems related to efficiency when multiple gateways are present. We discuss these issues at the end of this paper.

The rest of this paper is organized as follows: In section 2 we discuss some related work. Section 3 presents a description of the problem for both unicast and multicast routing. Section 4 describe our implementation of an ad hoc to terrestrial network gateway. Section 5 provides a discussion regarding how our work can be extended to the scenario of multiple gateways. Finally section 6 describes our conclusions and some directions for future work.

\section{Related Work}

There has been some work recently in addressing the problem of interfacing wireless ad hoc networks with terrestrial networks. Most of the work has focused on providing Internet connectivity to the ad hoc network. In particular, people have studied in some detail the use of mobileIP and registration based protocols to solve the problems of integrating the two networks.

[1] addresses the issue of providing global Internet access for MANETs, in particular, focusing on routing, the problem of global address resolution and gateway discovery. They provide an excellent discussion of the issues and an architecture for attempting to solve these problems. Similarly, [2] presents a scheme for providing Internet connectivity for ad hoc mobile networks. They provide a mechanism to enable cooperation between MobileIP and the AODV routing protocol. They use simulation results to validate their architecture by showing that it can maintain high throughput while keeping the overall control overhead low. [3] provides a brief description of how AODV can be used for providing inter-networking between wireless ad hoc networks and the IPv6 Internet. The primary focus is to describe the process of gateway discovery by using a multicast group, and the determination of a node address.

[4] differs from the above works in that it describes an actual software implementation of an integrated connectivity solution. In architecture, the solution described in [4] is 
similar to the solutions of [1] and [2], in that it combines the use of AODV in the wireless ad hoc network and MobileIP in the terrestrial network. They describe the implementation of gateway nodes that run both AODV and MobileIP software and are responsible for providing the connectivity.

While all the works described so far have focused on the use of AODV, [5] on the other hand proposes the use of a protocol independent gateway for ad hoc networks. This cluster gateway is responsible for providing Internet connectivity for the ad hoc network by acting as both a service access point as well as a MobileIP foreign agent. Ad hoc nodes register themselves with the cluster gateway, which makes its location known by periodic advertisements.

Although [6] uses ODMRP which has multicast routing capability, they focus on the unicast performance of ODMRP in an extended hybrid network consisting of both ad hoc and terrestrial nodes. They present an implementation of an extension of ODMRP which allows the ad hoc network to dynamically connect to the wired network. They do not, however, address how multicast operation can be achieved in the same scenario.

In [7] the authors examine in detail the use of MobileIP for providing Internet connectivity for Ad Hoc networks. They consider both proactive as well as reactive approaches to maintaining registration with the MobileIP foreign agent and present simulation results to show the benefits of their hybrid approach. They also do not consider multicast operation.

The solutions presented so far often involve complex protocols to achieve their design objectives. To the best of our knowledge, a unified gateway that supports both unicast as well as multicast routing between a terrestrial and an ad hoc routing protocol has not been implemented before.

\section{Problem Description}

The problem of providing connectivity between ad hoc and terrestrial routing protocols is becoming increasingly important. This connectivity problem can be divided into two parts: the first dealing with addressing unicast routing connectivity and the second addressing interoperation between multicast routing protocols in the two routing domains.

Numerous proposals have attempted to solve the first problem as described in the section 2, however the proposed solutions do not possess certain key characteristics. We attempted to base our solution on the following design principles:

- The solution should not add unnecessary complexity to an already complex problem.

- The solution should attempt to be as close in principle as possible to the way the problem of inter-domain routing is handled in the current Internet.

- The solution should be implementable in a realistic scenario.

- The solution should require only minimal or no modifications to terrestrial routing protocols. The terrestrial routing protocols are widely accepted standards, whereas the ad hoc routing protocols are still being developed.

- The solution should include support for both unicast as well as multicast routing protocols. 
In the following subsections we further characterize the two problem areas: of unicast and multicast routing gateways. The basic differences in the way these are implemented in the Internet motivates us to consider these as two separate problems. We later combine them into the same gateway implementation.

\subsection{Unicast Routing}

In AODV the establishment of a route is made on demand. This is in direct contrast to most proactive terrestrial routing protocols that assemble all possible routes as they learn of other hosts. In order to provide a native integration between ad hoc and terrestrial networks, it would be necessary for the gateway to determine the availability of individual hosts on a periodic basis so they can be advertised to the terrestrial domain.

The above problem is composed of two distinct parts. The first is how nodes from the ad hoc domain reach nodes in the terrestrial domain and the second is how nodes in the terrestrial domain reach nodes in the ad hoc network domain. Solving the first requires that nodes in the ad hoc domain be able to distinguish between destinations that are within the ad hoc domain and those that are outside. Attempting to solve the second problem requires that a mechanism exist for notifying the terrestrial domain routing protocol which nodes are present in the ad hoc domain.

Complex protocols and solution can be devised to solve each of these, however, we argue that if we adopt a single simplifying assumption the solutions become almost trivial. By representing the ad hoc network as a single aggregated address space we can easily solve the problem for routing traffic in and out it. Moreover, this approach also has the very attractive benefit of greatly simplifying the problems of address configuration and duplicate address resolution in the ad hoc network.

\subsection{Multicast Routing}

In multicast routing packets sent to a group are distributed to many hosts in multiple networks. In a terrestrial network a host subscribed to a multicast group need only notify its router of its interest in receiving traffic for that multicast group using IGMP. The routers construct a tree to distribute the packets based on which multicast routing protocol is being used. Therefore, IGMP is responsible for maintaining group membership information at the local network level while a multicast routing protocol such as MOSPF or DVMRP is responsible for the formation of the multicast forwarding tree by providing information to the routers in-between the source and destinations of the multicast traffic.

In ad hoc networks since each node is also a router IGMP is inappropriate because there is no real local network. Instead the operation of maintenance of group membership is entirely performed by the multicast routing protocol. This difference in philosophy in the two types of networks creates a problem when we try to implement a gateway. Terrestrial multicast routing protocols assume the presence of IGMP on all interfaces to notify them of group membership changes. Once again, our goal is seamless interoperation between the two domains such that nodes in the ad hoc domain are able to join multicast groups originating in the terrestrial domain and nodes in the terrestrial network are able to join multicast groups originating in the ad hoc network domain. 


\section{Ad Hoc to Terrestrial Network Gateway Description}

In this section we describe our implementation of a gateway between an ad hoc network and a terrestrial network. For our implementation we used OSPF as the terrestrial routing protocol and AODV as the ad hoc network routing protocol. We selected OSPF as a good candidate as at least one implementation which was publicly available included support for multicast operation as well. We used the OSPF implementation from www.ospf.org. As this version of OSPF includes support for multicast operation in this paper we use the terms OSPF and MOSPF interchangeably in this paper. For our ad hoc network routing protocol we used the AODV-UU version of AODV. We modified AODV-UU to include support for the multicast extensions to AODV. In this paper we use the terms AODV and MAODV interchangeably to refer to this implementation. Both our choices of routing protocols provided unicast and multicast routing support in one software distribution. This greatly simplifies the task of building a unified gateway. We constructed our gateway node to have a wired and a wireless interface. It runs both the MOSPF software as well as our version of MAODV routing protocol.

\subsection{Unicast Routing Gateway}

As described in an earlier section, the problem of implementing a unicast routing gateway involves being able to determine and distinguish between nodes that are within the ad hoc network and those that are outside. This can be accomplished via the use of complex registration and query protocols. However, if we make a single assumption that the ad hoc network can be represented as a single aggregated address space, we no longer need complex registration protocols.

To ensure interoperability between a terrestrial network and an ad hoc network, the ad hoc network must take care to ensure that its nodes select globally unique addresses. In the case where the terrestrial network is the Internet, the address space would need to be assigned via some body such as the IANA. Therefore, it is natural to assign a fixed prefix for the ad hoc network domain.

Using this assumption we can configure the gateway to advertise the ad hoc network as a prefix. This solves the problem of allowing terrestrial nodes to reach nodes within the ad hoc domain as packets destined for the ad hoc domain will automatically be routed to the gateway node which will be responsible for forwarding them into the ad hoc domain. In our implementation we configured OSPF to manage both the terrestrial and ad hoc network as OSPF areas. This isn't completely necessary: simply advertising the ad hoc network as an external route is just as functional. No changes needed to be made to the OSPF daemon.

For the ad hoc domain nodes to be able to reach nodes in the terrestrial domain is also trivial, as each node knows by simply looking at the destination whether that address is in the ad hoc or terrestrial domain. To reach external nodes, ad hoc nodes simply send a RREQ messages as usual. When these RREQ messages reach the gateway, it will reply with a RREP message if that destination is in the terrestrial network.

This method also has the advantage of simplicity. There is no need for the gateway router running OSPF to keep track of the dynamics of the ad hoc network. The gateway acts as shield by advertising a single aggregate route to the terrestrial domain. This protects the terrestrial network from the large numbers of updates that mobile ad hoc nodes might otherwise generate. 


\subsection{Multicast Routing Gateway}

Our approach to implementing the gateway between MOSPF and MAODV is similar to the approach we use for unicast routing: we make the ad hoc domain appear like a single network segment. MOSPF expects to hear IGMP messages on the wireless interface in order to forward multicast traffic onto it. We create a proxy agent on the gateway that performs the job of translating the MAODV group join/leave messages into appropriate IGMP messages and injects these messages into the IP stack for MOSPF to receive.

Therefore, for the multicast gateway it is necessary to configure MOSPF to treat the the ad hoc network as a full OSPF area. Since requests and joins will be coming in on the ad hoc interface, it is necessary for the MOSPF daemon to be listening on this interface to be aware of multicast activity in the ad hoc network. The MAODV daemon on the gateway is modified to join all multicast groups it hears about. This is important because the gateway has to be able to inform the terrestrial network about the presence of these groups.

An alternate solution would have been to modify MOSPF to listen to MAODV messages and process them as IGMP messages, but this would require extensive modifications to MOSPF. Our approach has the desired property that we do not need to modify MOSFP. The only changes we needed to make were to the MAODV code that ran on the gateway.

When a multicast group exists in the terrestrial network and a node in the ad hoc domain wishes to join it, the node sends out a RREQ for that group; when this reaches the gateway our modified MAODV code injects an IGMP group_join message into the IP stack such that the MOSPF deamon sees there is a node on the wireless interface network that wishes to subscribe to that group. MOSPF will then process this injected IGMP message and propagate this interest out into the terrestrial domain.

When a multicast group exists in the ad hoc network and a node in the terrestrial domain wishes to join this group, it will send out IGMP group_join messages which will propagate this interest through the terrestrial network until it reaches the gateway node. The gateway node, by virtue of being a part of the ad hoc domain, is already subscribed to this group and will simply start forwarding multicast traffic from the wireless ad hoc network interface onto the wired terrestrial interface.

\subsection{Implementation Details and Testbed Description}

We implemented our gateway on nodes that ran Red Hat Linux 7.3 with a Linux 2.4.19 kernel from www.kernel.org. OSPFD version 2.0 from ospf.org was used as the terrestrial routing daemon and AODV-UU version 0.6 was used as the AODV implementation (user.it.uu.se/ henrikl/aodv/). Multicast additions to AODV-UU made by our group were applied to the source. In addition, the code was further modified to support the gateway operation as described in the previous sections. No changes were made to the MOSPF software.

We only needed to make minor modifications to AODV-UU to support correct operation as a unicast gateway. AODV-UU has a basic gateway mode where the configured host will automatically offer a default route for destinations off the network. In the version of AODV-UU we used (version 0.6), this mode did not properly function and was revised. One of the major changes was a fix to make the AODV daemon check the kernel's route table and only return a reply if an appropriate route was found. 


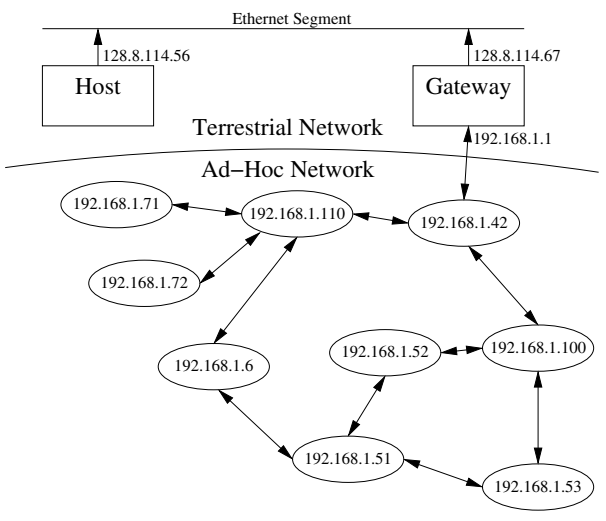

Fig. 1. Gateway Test Network

Modifying MAODV to support the multicast gateway capability, required significantly more work. Changes were made such that the gateway would join groups on reception of a group hello message and remove itself only once all upstream and downstream hosts have removed themselves. In addition, we needed to mimic the behavior of IGMP on the wireless interface of the gateway. A kernel module was created to inject packets into the IP stack. This module provides a directory in /proc/net with a node for each network device. A write to one of these nodes is converted into a single incoming packet. We then used this interface to inject IGMP packets into the IP stack.

Our test network topology is shown in Figure 1. We used appropriate subsets of this topology to test different functions of the gateway. We tested unicast connectivity in our testbed using a simple ping as well as tcpdump to analyze the traffic being received and forwarded on various segments to insure proper behavior. It was particularly important to ensure correct operation of the gateway when the ad hoc network contains nodes that are multiple hops away from the gateway. We were able to verify correct operation of the gateway in this scenario as well. Multicast connectivity was initially tested with a simple custom program to join a group and periodically transmit and receive multicast packets. In addition we also used vic, a common multicast video streaming application, to verify correct multicast connectivity During these tests, both unicast and multicast route/forwarding tables were monitored to verify the proper behavior of the routing protocols.

\section{Multiple Gateways Scenario}

Until now we have focused our attention on the problem where the ad hoc and the terrestrial network have a single point of attachment. Next we turn to more complex scenarios where there are multiple points of attachment. In this section we illustrate some of the issues that arise when we take into consideration the possibility of multiple gateways and discuss some potential solutions to these problems. 


\subsection{Multiple Unicast/Multicast Gateways}

There are several problems that arise in unicast routing protocol interoperability when we allow for scenarios where multiple gateways can exist. Some are related with with interaction between the nature of proactive and reactive unicast routing protocols, and some are particular to multicast routing. The problem with unicast routing are related to the inability of the gateways to obtain complete information regarding the availability and location of different nodes in the ad hoc network. This leads to inefficient routing and reachability problems when the network is partitioned. Multicast routing protocol interoperation in the presence of multiple gateways is difficult because group membership management is even further complicated.

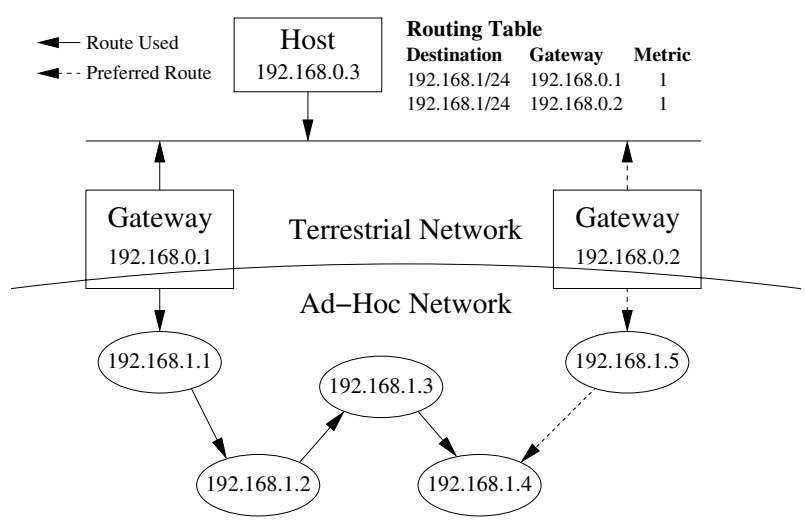

Fig. 2. Example of Poor Route Selection

1. Inefficient routing paths: This problem is illustrated in Figure 2. The ad hoc network can be reached via either gateway; however, the metrics that are advertised into the terrestrial network only reflect the reachability of the gateway in the terrestrial domain and do not reflect the number of hops inside the ad hoc network. This can result in inefficient paths being chosen from nodes in the terrestrial domain to the ad hoc domain. The decision of which gateway the terrestrial nodes choose is determined only by their distance to the gateway and not the total distance to the destination. It might have been possible to reach the destination node via fewer hops by choosing a gateway that was slightly further away in the terrestrial domain but closer to the final destination node in the ad hoc network. This problem does not exist for nodes in the ad hoc network attempting to reach nodes in the terrestrial domain, as the RREP messages that the gateways generate reflect the correct metrics of the node in the terrestrial network.

2. Ad Hoc Network Partitions: The gateway nodes only advertise the reachability of the ad hoc network, not that of individual nodes. When a partition occurs in the ad hoc network, all gateways will not be able to reach all nodes. This can result in a situation where traffic originating from the terrestrial network can get routed to a 


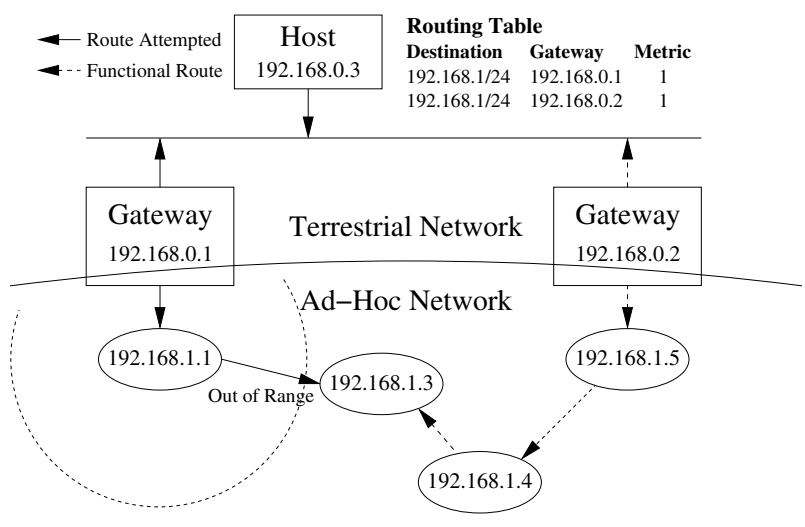

Fig. 3. Example of Unnecessary Failure due to Partition

gateway which is unable to reach a particular node even though that node might be reachable via another gateway. This problem is depicted in Figure 3 .

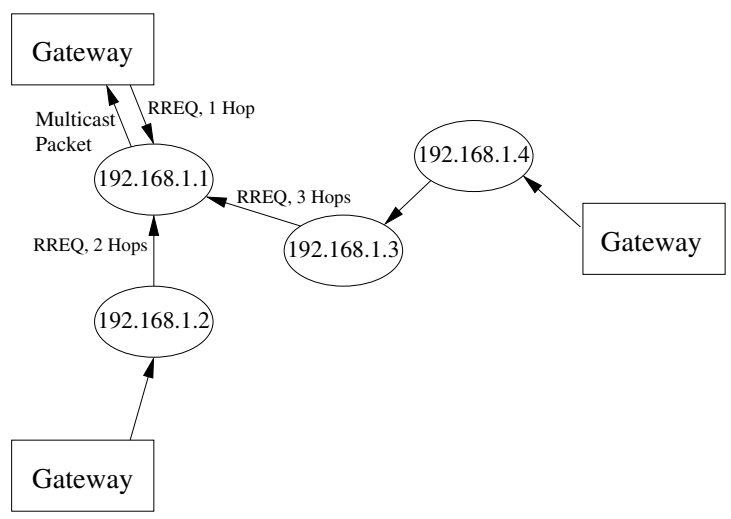

Fig. 4. Multicasting to a Non-existent Group

3. Multicasting to a non-existent group from the ad hoc domain. If a host wishes to message a group without joining the group using MAODV, it will send a RREQ without the join flag set. Several gateways will reply to this RREQ however, the host will pick only one path to the nearest gateway. This can create a problem as only the terrestrial network connected with that gateway will receive the muticast packets. This is shown in Figure 4

4. Inability to detect group prunes. In order to conserve resources, it is desirable that gateways will prune groups that are no longer active. This can fail once multiple gateways have joined the group as shown in Figure 5. If there is only one gateway on the network, when the last interested member leaves the group, all intervening nodes between the gateway and that node will prune themselves. When multiple 


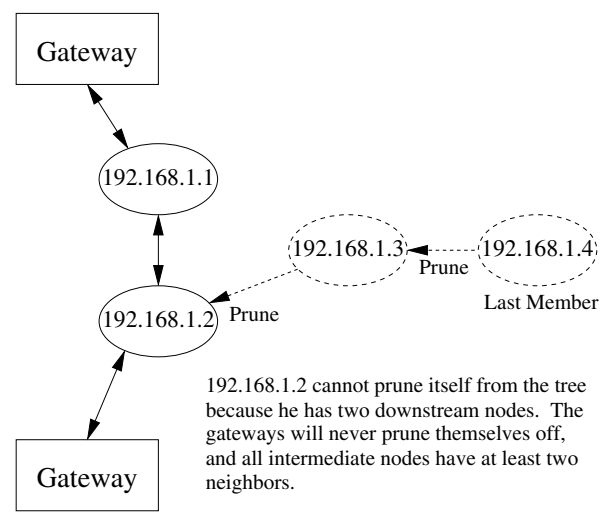

Fig. 5. Example of Inability to Prune Inactive Groups

gateways are in the group, atleast one node will have two active downstream nodes when the last member leaves, as shown in Figure 5. This node will be unable to prune itself and the group will persist despite having no active members.

\subsection{Discussion}

The problems described in the previous section related with unicast routing are a direct result of our attempts to insulate the terrestrial network from the frequent changes in the ad hoc network. There is no single solution for all these problems. One approach might be to use a registration based protocol to notify each gateway about nodes in the ad hoc network that it is responsible for. Another might be the use of a proactive protocol in the ad hoc domain, but this has its own drawbacks.

The problem with multicast routing are equally difficult. If one aggressively prunes idle groups in an attempt to recover resources, broadcasts from non-members to offnetwork groups may be lost. On the other hand, if one forms groups for every route request, many idle groups are created that will not be pruned. A modification to the multicast routing protocol could be added to allow gateways to test for the presence of completely inactive groups, but modifications to the protocol and all supporting software would be undesirable for many applications. Another solution would be a compromise between resource consumption and reliability of non-member multicasts. The gateways would have a soft-limit on number of active multicast groups. Once this limit is reached, idle groups begin to be pruned. As the number of groups approaches a hard-limit, the aggressiveness of the prune increases.

In summary, the use of multiple gateways between the same terrestrial and ad hoc networks can create scenarios where efficiency and even accuracy of the gateway is reduced. Therefore, we recommend that, care should be taken to ensure the proper functioning of the integrated network when multiple gateways are present, and scenarios such as the ones we have described should be avoided. 


\section{Conclusions and Future Work}

In this paper we have presented an implementation of a gateway to provide interoperability between routing protocols in the ad hoc and terrestrial network domains. We have provided details of our design and our implementation. We hope that this will provide valuable information to other developers of routing gateways. While our solution works for this particular set of routing protocols, we are investigating how we can make our implementation more generic so that it is easy to implement the same functionality for any set of terrestrial and ad hoc routing protocols. In addition, we also discussed some issues related to realise internetworking in the presence of multiple terrestrial to ad hoc network gateways. We argue that due to the additional complexity introduced by multiple gateways, care should be taken to ensure the proper functioning of the integrated network in the presence of multiple gateways or only a single active gateway between the networks should be permitted.

We are currently attempting to validate our gateway approach on a network using a combination of RIP and DVMRP instead of MOSPF. In addition we are investigating ways of resolving the issues and improving the performance of our solution for scenarios where multiple gateways are present.

Acknowledgments. The material described in this paper is based upon work performed in collaboration with Telcordia Technologies and sponsored by the U.S. Army Research Lab. award number DAAD17-00-C-0115. The views and conclusions contained in this document are those of the authors and should not be interpreted as representing the official policies either expressed or implied of the Army Research Lab or the U.S. Government.

\section{References}

1. B. Andreadis. Providing Internet Access to Mobile Ad Hoc Networks. London Communications Symposium, Sep 2002.

2. Y. Sun, E. M. Belding-Royer, and C. E. Perkins. Internet Connectivity for Ad Hoc Mobile Networks. International Journal of Wireless Information Networks, Apr 2002.

3. A. Nilsson, C. Perkins, R. Tuominen, A. J. Wakikawa, and Malinen J. T. AODV and IPv6 Internet Access for Ad Hoc Networks. Mobile Computing and Communications Review, Vol. 6, Number 3, Jul 2002.

4. C. Ahlund and A. Zaslavsky. Integration of Ad hoc Network and IP Network Capabilities for Mobile Hosts. 10the International Conference on Telecommunications (ICT), Feb 2003.

5. A. Striegel, R. Ramanujan, and J. Bonney. A Protocol Independent Internet Gateway for AdHoc Wireless Networks. Proceedings of Local Computer Networks (LCN) 2001, Nov 2001.

6. S. H. Bae, S. Lee, and M. Gerla. Unicast Performance Analysis of Extended ODMRP in a Wiredto-Wireless Hybrid Ad Hoc Network. IEEE International Conference on Communications and Networks (ICCN), Las Vegas, Oct 2000.

7. P. Ratanchandani and R. Kravets. A Hybrid Approach to Internet Connectivity for Mobile Ad Hoc Networks. Proceedings of IEEE Wireless Communications and Networking Conference $(W C N C)$, Mar 2003. 